\title{
Is dark energy from cosmic Hawking radiation?
}

\author{
Jae-Weon Lee* \\ School of Computational Sciences, Korea Institute for Advanced Study, \\ 207-43 Cheongnyangni 2-dong, Dongdaemun-gu, Seoul 130-012, Korea and \\ Department of energy resources development, Jungwon University, \\ 5 dongburi, Goesan-eup, Goesan-gun Chungbuk Korea 367-805 \\ Hyeong-Chan Kim丹 \\ Center for Quantum Spacetime, Sogang University, Seoul 121-742, Republic of Korea \\ Jungjai Lef团 \\ Department of Physics, Daejin University, Pocheon, Gyeonggi 487-711, Korea
}

(Dated: October 27, 2018)

\begin{abstract}
It is suggested that dark energy is the energy of the Hawking radiation from a cosmic horizon. Despite of its extremely low Gibbons-Hawking temperature, this radiation could have the appropriate magnitude $O\left(M_{P}^{2} H^{2}\right)$ and the equation of state to explain the observed cosmological data if there is a Planck scale UV-cutoff, where $H$ is the Hubble parameter.
\end{abstract}

PACS numbers: 98.80.Cq, 98.80.Es, 03.65.Ud

\section{INTRODUCTION}

In this paper, we suggest that the dark energy is the energy of the Hawking radiation from a cosmic horizon. The ' dark energy having positive energy density $\rho_{\Lambda}$, negative pressure $p_{\Lambda}$ and the equation of state $w_{\Lambda} \equiv p_{\Lambda} / \rho_{\Lambda}$ smaller than $-1 / 3$ seems to cause the accelerating expansion of the universe which can be inferred by the observations of the ' type Ia supernova [1, 2], the cosmic microwave background radiation (CMBR) 3] and the large scale structures [4]. ' The exact origin of this mysterious dark energy is one of the hardest puzzles in modern physics and astronomy [5]. The dark energy problem consists of three sub-problems; why the dark energy density is so small, nonzero, and comparable to the critical density at the present. Although, there are already various models relying on materials ' such as quintessence [6, 7], $k$-essence [8], phantom [9, 10], Chaplygin gas [11], and quintom [12] among many [13, 14], no model has solved the problems in a natural manner so far. The holographic dark energy (HDE) models [15, 16] are based on the holographic principle saying the number of degrees of freedom inside a volume is proportional to ' the surface area of the region [17] rather than the volume. The connection between dark energy and the holographic principle implies that dark energy could be related to a cosmic horizon at which the principle applied. The energy ' density of HDE is usually $O\left(H^{2} M_{P}^{2}\right)$, which is similar to the observed value, where $H$ is the Hubble parameter. ' This form of dark energy is also often found in many dark energy models based on the quantum vacuum fluctuation ' $18,19,20,21,22,23,24,25,26,27,28]$. However, it is still uncertain why the dark energy should be related to a cosmic horizon in this form and why a horizon property is related to dark energy density at the center of the horizon.

Recently, we have suggested a new idea that dark energy is related to the entanglement of the quantum vacuum fluctuation [29] or the erasure of the quantum information of the vacuum at the cosmic horizon [30]. This model predicts $\rho_{\Lambda}$ and $w_{\Lambda}$ consistent with the observational data. According to the model, the entanglement of virtual particles around the expanding cosmic event horizon induces a kind of thermal energy which can be interpreted as dark energy. (This idea is followed by related works [31, 32, 33].) One possible interpretation for this entanglement dark energy is that dark energy is just the energy related to the cosmic Hawking radiation or Unruh radiation [34, 35]. According to Hawking, in the black hole physics, the entropy of Hawking radiation can be identified with the entanglement entropy between the outgoing and incoming particles created at the black hole horizon due to the quantum fluctuation [36, 37]. One can imagine that a similar thing happens at a cosmic horizon and dark energy is the energy of this cosmic Hawking radiation.

In this paper, we investigate in detail the possibility that the dark energy is the energy of Hawking radiation itself

\footnotetext{
*Electronic address: scikid@gmail.com

$\dagger$ †lectronic address: hyeongchan@gmail.com

${ }^{\ddagger}$ Electronic address: jjlee@daejin.ac.kr
} 
from the cosmic horizon. In Sec. II we discuss the quantum nature of this dark energy. In Sec. III we show the relation between the entropy of the cosmic Hawking radiation and dark energy. In Sec. VI the predictions of our theory are compared with the observational data and cosmological constraints. Section V contains discussions.

\section{QUANTUM NATURE OF DARK ENERGY}

At a first glance, the idea linking dark energy to the Hawking radiation may look counterintuitive, because the Gibbons-Hawking temperature of the present cosmic event horizon $T_{H} \sim O(H) \sim 10^{-60} M_{P} \simeq 10^{-32} \mathrm{eV}[38]$ is extremely low and thermal radiation usually has a positive equation of state unlike dark energy. However, it is known [39] that Hawking radiation and ordinary thermal radiation such as CMBR are very different in their nature. The ordinary radiation is composed of excited quanta of which existence usually has an observer independent meaning, while the presence of the Hawking radiation, being the transformed vacua, is observer dependent [40]. For example, an observer at rest far from a black hole can see the Hawking radiation from the horizon, while another observer freely falling toward the black hole could not see the radiation. Similarly, the cosmic Hawking radiation seen by one observer $O_{A}$ could be just the vacuum fluctuation for another observer $O_{B}$ sitting at a different position. (See Fig. 1.) Instead, the observer $O_{B}$ could see his/her own Hawking radiation from his/her own horizon.

The quantum field theoretic calculations of the energy density of the cosmic Hawking radiation support this idea too. It is well known that the cosmic Hawking radiation in de Sitter universe has a energy momentum tensor $T_{\mu \nu}=\rho g_{\mu \nu}$ and hence the equation of state -1 like the cosmological constant [39] which is different from that of the thermal radiation. Unfortunately, this Hawking radiation is known to have too small energy density [39],

$$
\rho=\left\langle T_{00}\right\rangle \sim\left\langle\phi^{2}\right\rangle \sim \int^{\infty} k^{2} \phi_{k}^{2} d k \sim H^{4}
$$

to explain the observed dark energy density and, hence, it has never been considered as a candidate for dark energy. This value was obtained by calculating Bunch-Davies vacuum energy density for a scalar field $\phi$ in de Sitter universe [41]. Here, $\phi_{k}$ is the mode with the momentum $k$ on the curved space-time, which contains $H$ in its expression. However, this value was obtained by using the renormalization technique with an infinite UV cut-off and not adequate when there is a finite UV cut-off $k_{u}$, as in our case. It was shown in [42] that with a finite UV-cutoff $k_{u}$, the energy density above should be changed to

$$
\rho \sim \int^{k_{u}} k^{2} \phi_{k}^{2} d k \sim H^{2} k_{u}^{2}
$$

We notice that if we choose a natural value, $M_{P}$, as the UV-cutoff, the energy density of Hawking radiation is not $O\left(H^{4}\right)$ but $O\left(H^{2} M_{P}^{2}\right)$, which is just of order of the dark energy density observed! Thus, for a De Sitter-like universe, we can expect that the cosmic Hawking radiation is a plausible candidate for dark energy.

\section{DARK ENERGY AND COSMIC HAWKING RADIATION}

Let us roughly estimate this dark energy (Hawking radiation energy) density by assuming that the change of Hawking radiation energy is given by the change of "horizon energy" [43]

$$
d E_{\Lambda}=T_{H} d S_{\Lambda}
$$

where $T_{H}$ is the temperature of the horizon. In [29] we use this usual 'first law-like equation' to define the entanglement energy. The relation between this energy and causal horizons is investigated by Jacobson [44]. We are supposing that the emission of the cosmic Hawking radiation actually represents the change of the vacuum energy or the horizon energy satisfying Eq. (3) as in black hole cases.

The Hawking radiation for an observer could be just the vacuum state for another observer. In cosmology, the Hubble horizon, the event horizon, and the apparent horizon are usually considered for the comic horizon. Let us denote the radius of these generic horizons with $r$. For the cosmic horizons the Hawking temperature is usually given by $T_{H} \sim 1 / r$ [45, 46] and is of order the Hubble parameter $H$. Thus, the typical dark energy from the cosmic Hawking radiation is $E_{\Lambda}=\int d E_{\Lambda} \sim \int T_{H} d S_{\Lambda} \sim M_{P}^{2} r$ and its energy density is $\rho_{\Lambda}=3 E_{\Lambda} / 4 \pi r^{3} \sim M_{P}^{2} / r^{2} \sim M_{P}^{2} H^{2}$ which is the value just required. Here, inspired by the holographic principle, the dark entropy $S_{\Lambda}$ is assumed to be proportional to the horizon area in Planck unit, i.e., $S_{\Lambda} \sim M_{P}^{2} r^{2}$. This 'area-law' for the entropy related to the horizon is typically observed in the entanglement theory [47], string/M theory [48] and the quantum gravity theory [17]. (One 
can say that the Hawking radiation has a 'surface' property while the CMBR has a 'volume' property, which is the key property allowing the Hawking radiation to be a plausible dark energy candidate.) Therefore, this kind of dark energy generally has the form of HDE and could explain the present value of the observed dark energy density. Although the HDE model is a promising alternative, it has its own difficulties [49, 50, 51] and the exact origin of the HDE itself has yet to be adequately justified in the field theoretic context.

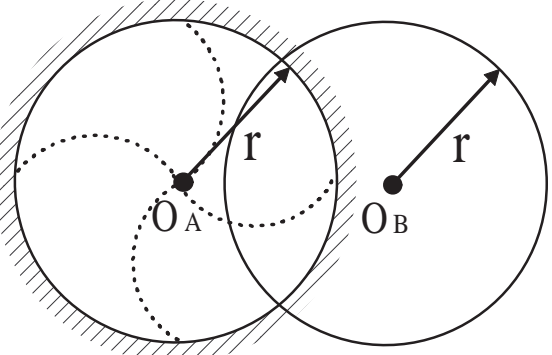

FIG. 1: Hawking radiation (dotted lines) from a cosmic horizon with a radius $r$ can play a role of dark energy. The radiation seen by one observer $O_{A}$ could be the vacuum fluctuation for another observer $O_{B}$ sitting at a different position. Instead, the observer $O_{B}$ could see his/her own Hawking radiation from his/her own horizon.

It is also worth mentioning that the cosmic Hawking radiation and Hawking radiation from a black hole have differences too. For the black hole, the Hawking temperature increases as the Hawking radiation is emitted, while for the universe the temperature decreases. This may have a quantum informational origin [29, 30]. In the cosmological case, the outside of the horizon is invisible to the observer, while for the black hole the inside of the horizon is invisible. We have seen that if the entropy of the cosmic Hawking radiation satisfies the 'area-law', it is almost unavoidable that the radiation behaves as dark energy. The key issue now is how much entropy cosmic Hawking radiation actually has.

How can we explicitly calculate the entropy of the cosmic Hawking radiation? Hawking et al. [52], argued that the entropy of the de Sitter horizon can be described as the quantum entanglement of the conformal field theory vacuum across the horizon and it can also be viewed as the entropy of the thermal Rindler particles near the horizon, i.e., Hawking radiation. In this paper we follow this interpretation. They also showed that the entanglement entropy of four-dimensional space-time with a horizon such as the de Sitter universe is

$$
S_{E n t}=\frac{A_{4}}{4 G_{4}}=\frac{2 N_{d o f} A_{4}}{a^{2} \pi},
$$

where $a$ is a UV cut-off, $G_{4}$ is the 4-dimensional gravitational constant, $N_{\text {dof }}$ is the degrees of freedom, and $A_{4}$ is the area of the horizon. Thus, the 4-dimensional Planck mass is related to $G_{4}$ and the number of fields in this theory.

Similarly, one can also calculate the entropy of the Hawking radiation using the concept of entanglement of quantum fields [53, 54]. The entanglement entropy of a quantum field with a horizon is generally expressed in the form $S_{E n t}=\beta r^{2} / a^{2}$, where $\beta$ is an $O(1)$ constant that depends on the nature of the field. Entanglement entropy for a single massless scalar field in curved backgrounds with a time-dependent event horizons is calculated in Ref. [53, 54] using a Hamiltonian approach. By performing numerical calculations on a sphere lattice, $\beta=0.30$ was obtained for Friedmann-Robertson-Walker universe. If there are $N_{\text {dof }}$ spin degrees of freedom of quantum fields within $r$, due to the additivity of the entanglement entropy [55], we must add up the contributions from all of the individual fields to $S_{E n t}$ [53], that is, $S_{E n t}=N_{d o f} \beta r^{2} / a^{2}$. In this case dark energy becomes the entanglement dark energy in 29].

To explicitly calculate the dark energy density, let us choose a specific horizon for the dark energy. It is well known that HDE with the particle horizon does not give an accelerating universe [56], while HDE with the event horizon does [15]. Thus, henceforth, we investigate the most plausible case, where $r=R_{h}$ is the radius of the future event horizon

$$
R_{h} \equiv R(t) \int_{t}^{\infty} \frac{d t^{\prime}}{R\left(t^{\prime}\right)}=R(t) \int_{R}^{\infty} \frac{d R\left(t^{\prime}\right)}{H\left(t^{\prime}\right) R\left(t^{\prime}\right)^{2}}
$$

For the temperature $T_{H}$ of the Hawking radiation we use the Gibbons-Hawking temperature [40] $1 / 2 \pi R_{h}$ and for the entropy $S_{\Lambda}$ we choose the entropy satisfying the holographic principle, i.e, $S_{\Lambda}=\pi R_{h}^{2} m_{P}^{2}$. This choice of $S_{\Lambda}$ corresponds to the first equality of Eq. (4). This is a plausible choice because our universe is going to a dark energy dominated universe, which can be a quasi-de Sitter universe [57]. Here $m_{P}=\sqrt{8 \pi} M_{P}$ and we consider the flat $(k=0)$ Friedmann universe described by the metric $d s^{2}=-d t^{2}+R^{2}(t) d \Omega^{2}$. By integrating Eq. (3) one can easily obtain 
$E_{\Lambda}=8 \pi R_{h} M_{P}^{2}$ and $[15$

$$
\rho_{\Lambda}=\frac{3 E_{\Lambda}}{4 \pi R_{h}^{2}}=\frac{3 d^{2} M_{P}^{2}}{R_{h}^{2}}
$$

with the parameter $d=\sqrt{2}$. This $\rho_{\Lambda}$ decreases much slowly than $\rho_{r}$ and could eventually dominate the universe [38].

Alternatively, considering that the temperature is varying, one may use the minimum free energy condition [30] $d F=d\left(E_{\Lambda}-T_{H} S_{\Lambda}\right)=0$ instead of Eq. (3), or,

$$
d E_{\Lambda}=d\left(T_{H} S_{\Lambda}\right)
$$

which leads to $d=1$. The current observational constraint is $d=0.91_{-0.18}^{+0.26}$ [58], which is in a good agreement with our predictions. Since our universe is not exactly equal to the de Sitter universe, $S_{\Lambda}$ or $T_{H}$ can be slightly different from that of the de Sitter universe. Thus, it is expected that $d$ is approximately 1 as observed.

\section{OBSERVATIONAL CONSTRAINTS}

Let us move on the issue of the equation of state, or how the cosmic Hawking radiation could accelerate the expansion of the universe. Recall that the Hawking radiation, even when it is the electromagnetic waves, does not have the equation of state for thermal radiation, $1 / 3$. It is important to note that, contrary to intuition, the (effective) equation of state of matter in the universe is a dynamical property rather than its intrinsic nature. It depends on how the energy density changes as the universe expands [29]. (See Eq. [8].) For example a scalar field rolling down very slowly its potential behaves like dark energy (i.e., quintessence), while during a rapid oscillation around the potential minima it behaves like ordinary matter.

To be more precise, consider perfect fluid having the energy momentum tensor $T_{\mu \nu}=\left(\rho_{\Lambda}+p_{\Lambda}\right) U_{\mu} U_{\nu}-p_{\Lambda} g_{\mu \nu}$, which the homogeneous dark energy should satisfies, where $U^{\mu} U_{\mu}=1$. It is well known that, from the cosmological energy-momentum conservation equation $T_{; \nu}^{\mu \nu}=0$, one can obtain

$$
p_{\Lambda}=\frac{d\left(R^{3} \rho_{\Lambda}\right)}{-3 R^{2} d R}
$$

This equation implies that the perfect fluid with increasing total energy within a comoving volume as the universe expands has effective negative pressure and could play a role of dark energy [38]. When HDE dominates other matter density, its energy density behaves as $\rho_{\Lambda} \sim R^{-2+2 / d}$ [15], hence, the total dark energy in the comoving volume $\rho_{\Lambda} R^{3}$ is an increasing function of time. Thus, the Hawking radiation having the energy in the form of HDE could act as dark energy for $d>0$, because its energy density $\rho_{\Lambda}$ decreases slower than $1 / R^{3}$.

Interestingly, our theory can be verified even current observational data. From Eq. (8) one can obtain the equation of state for our dark energy in the form of HDE in Eq. (6) [15, 59]

$$
w_{\Lambda}^{0}=-\frac{1}{3}\left(1+\frac{2 \sqrt{\Omega_{\Lambda}^{0}}}{d}\right),
$$

and the change rate of it at the present time [15, 60]

$$
\begin{aligned}
w_{1} & \left.\equiv \frac{d w_{\Lambda}(z)}{d z}\right|_{z=0} \\
& =\frac{\sqrt{\Omega_{\Lambda}^{0}}\left(1-\Omega_{\Lambda}^{0}\right)}{3 d}\left(1+\frac{2 \sqrt{\Omega_{\Lambda}^{0}}}{d}\right)
\end{aligned}
$$

where $z$ is the redshift parameter, $w_{\Lambda}(z) \simeq w_{\Lambda}^{0}+w_{1}(1-R)$, and the observed present value of the density parameter of the dark energy $\Omega_{\Lambda}^{0} \simeq 0.73$. For $d=1$ these equations give $w_{\Lambda}^{0}=-0.903$ and $w_{1}=0.208$, while for $d=\sqrt{2}$, $w_{\Lambda}^{0}=-0.736$ and $w_{1}=0.12$. The predictions of our theory well agree with the recent observational data; $w_{\Lambda}^{0}=$ $-1.03 \pm 0.15$ and $w_{1}=0.405_{-0.587}^{+0.562}[61$, 62]. If we interpret the entanglement dark energy in [29] as the energy of the cosmic Hawking radiation, then $w_{\Lambda}^{0}=-0.93$ and $w_{1}=0.11$. (See Fig. 2.) Thus, the cosmic Hawking radiation can give the appropriate equation of state for the observed dark energy, because the Hawking radiation gives the HDE with $\rho_{\Lambda}$ in Eq. (6). 


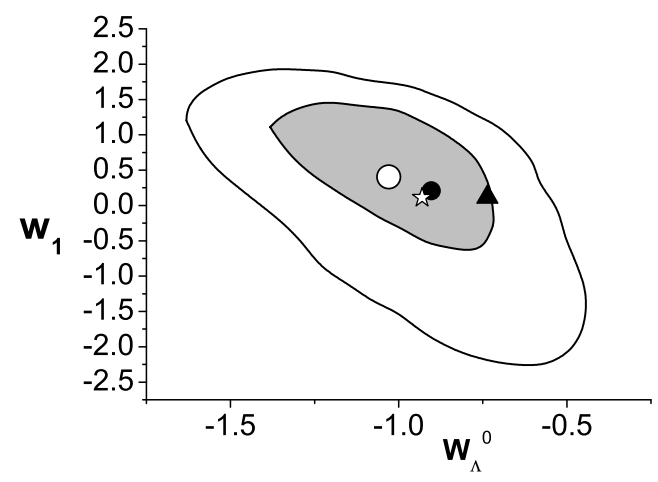

FIG. 2: Observational constraints on the equation of state parameters $w_{\Lambda}^{0}$ and $w_{1}$ (Fig. 2 of [61]) and our theoretical predictions. The contours represent $95 \%$ and $68 \%$ C.L., respectively. The white dot denotes the best-fit point from the observations. The black dot represents our theoretical prediction with $d E_{\Lambda}=d\left(T_{H} S_{\Lambda}\right)$ and the triangle with $d E_{\Lambda}=T_{H} d S_{\Lambda}$. The white star indicates the results for the entanglement dark energy in [29].

It was also shown that the dark energy in this form can solve the cosmic coincidence problem too, if there was an ordinary inflation with the number of e-fold $N \simeq 65$ at the very early universe [15, 38]. As is well known, to solve many problems of the standard big-bang cosmology we need an inflation $N \gtrsim 60$. Note that there is also a conjectured theoretical upper bound, $N \lesssim 65$ from the holographic principle [63, 64, 65] for the asymptotic de Sitter universe or from the density perturbation generation [66, 67]. Thus, very interestingly, the required $N$ value falls within the narrow range expected from other cosmological constraints. It is also interesting that the HDE model is connected to the second law of thermodynamics [68].

Now, we investigate whether the cosmic Hawking radiation is consistent with other cosmological constraints. The typical (peak) wavelength of the cosmic Hawking radiation at present is $\lambda \sim 1 / T_{H} \sim 1 / H$, which is comparable to the size of the observable universe. This huge wavelength explains why we could not detect dark energy directly so far and why dark energy seems to be so homogenous. For example, let us consider the decoupling of electron from the cosmic Hawking radiation. As is well known the electron is decoupled from the radiation when the scattering rate $\Gamma=n_{e} \sigma_{T}$ is smaller than the Hubble parameter $H$. Here, $n_{e}$ is the free electron number density, and $\sigma_{T}=6.65 \times 10^{-25} \mathrm{~cm}^{2}$ is the Thomson cross section [69]. Since the binding energy of hydrogen, $13.6 \mathrm{eV}$, is much larger than $T_{H}$, the ionization of hydrogen and the increase of $n_{e}$ due to the Hawking radiation is negligible and hence we can ignore the electromagnetic effect of the Hawking radiation on the hydrogen as long as $T_{H} \ll 13.6 \mathrm{eV}$. Another example is the effect of photons in the Hawking radiation on the ultra high energy cosmic ray (UHECR, mainly proton). In this case we can obtain Greisen-Zatsepin-Kuzmin (GZK)-like cut-off [70] for the energy of cosmic rays interacting with the Hawking radiation [71, 72, 73]

$$
E_{p}<\frac{m_{\pi}}{4 T_{H}}\left(2 m_{p}+m_{\pi}\right) \simeq 6.7 \times 10^{39} \mathrm{GeV}
$$

where, we used $T_{H}=10^{-32} \mathrm{eV}$ for the photon energy instead of the energy of usual CMBR photon, and $m_{p}$ is the proton mass and $m_{\pi}$ is the pion mass. This bound is well above the Planck energy and the observed constraints $E_{p}<6 \times 10^{10} \mathrm{GeV}$ [74]. Thus, the effect of the Hawking radiation photons on the UHECR is negligible compared to that of CMBR too. Furthermore, the temperature of the cosmic Hawking radiation is too low to distort the CMBR signal [75].

The cosmological principle implies the homogeneity of the Hawking radiation and this also justifies the simpleminded use of the spherical volume $4 \pi R_{h}^{3} / 3$ during the above calculation of $\rho_{\Lambda}$ at the center of the horizon sphere. Hawking radiation also provides a mechanism linking the local dark energy density to the global property of the horizon, because the gravitational field at the center plays a role of a detector for the energy density of the radiation from the horizon.

This dark energy neither spoils the standard inflation scenario. Note that this dark energy is different from the energy from quantum fluctuation of an inflation field $\phi$, even though the both energy is related to the quantum fluctuation of the vacuum. The inflaton energy density fluctuation comes from the fluctuation of the arrival time for the inflaton to roll down to the potential minima due to the quantum fluctuation $\delta \phi$ of the inflaton field. Thus, it is highly dependent on the shape of the potential. On the other hand, the energy of Hawking radiation is from all 
quantum fields regardless of its potential. Furthermore, in [38], it is shown that the size of the event horizon is an exponentially increasing function of time $t$ during the inflation, i.e.,

$$
R_{h}(t) \simeq \frac{1}{H_{i}}\left(1+A e^{H_{i}\left(t-t_{i}\right)}\right)
$$

where $\mathrm{A}$ and $t_{i}$ are constants and $H_{i}$ is the Hubble parameter at the inflation. Thus, after some e-folds of expansion, the energy density of Hawking radiation which is proportional to $1 / R_{h}^{2}$ is negligible compared to the inflaton energy density and does not spoil the usual scenario of inflation.

Although, $\rho_{\Lambda}$ has no $\hbar$ explicitly, the quantumness of the Hawking radiation can be seen clearly from the existence of $\hbar$ in Gibbons-Hawking temperature $T_{H}=\hbar / 2 \pi R_{h}$, where $R_{h}$ is the radius of the cosmic event horizon. Does the quantum nature of Hawking radiation spoil the classical description of back ground geometry? To see this is not the case, consider the back-reaction of the quantum fluctuation in chaotic inflation as an example. This is a second order effect in the cosmological perturbation. The fractional contribution of scalar metric perturbations $\rho_{s}$ to the total energy density $\rho_{0}$ is shown [76] to be

$$
\frac{\rho_{s}}{\rho_{0}} \sim-\frac{m^{2} \phi^{2}}{M_{P}^{4}}
$$

which is negligible when $\phi \lesssim M_{P}$ and the inflaton mass $m \ll M_{P}$. The energy scale of dark energy today is even much lower than this inflation scale. Furthermore, to make the local space-time fluctuate significantly due to the quantum effect the energy density of dominant matter should be at least $O\left(M_{P}^{4}\right)$ [77]. Thus, generally, the quantum fluctuation of the space-time due to the Hawking radiation is small compared to the quantum gravitational scale, and we can treat the evolution of the universe classically even when the dark energy from Hawking radiation dominates the universe. The cosmological effect of the Hawking radiation is relevant only at the cosmological scale in an average sense.

\section{DISCUSSION}

We can summarize how the cosmic Hawking radiation could solve the dark energy problem. In this scenario, the dark energy density is small due to the holographic principle, comparable to the current critical density because the number of e-folds during the inflation $N \simeq 65$ or the horizon size is $O(1 / H)$, and non-zero due to the unavoidable quantum fluctuation. The cosmic Hawking radiation also explains why dark energy is so homogeneous and can hardly interact with ordinary matter. Its wavelength is simply too long.

Let us discuss the possible direction of future research. Although the event horizon is the most natural candidate for our purpose from the viewpoint of quantum information science and gravity, it is still possible that the proper horizon for dark energy could be other horizon besides the event horizon [78] such as the apparent horizon [79]. In this case the interaction between dark matter and Hawking radiation would be important. Since $\omega_{\Lambda}^{0}$ and $\omega_{1}$ will be precisely constrained by observations in the near future, our model can be verified soon, once we derive the properties of the cosmic Hawking radiation more precisely. Thus, deriving the exact entropy or energy of the cosmic Hawking radiation would be of great importance in this direction. The temperature of the cosmic Hawking radiation may be too low to be detected above the CMBR 75], however, the cosmic Hawking radiation and its energy may be simulated using the acoustic horizons [80, 81] or optical black holes [82] in laboratories. If dark energy is really the energy of the cosmic Hawking radiation, this model provides not only a new direction to solve the dark energy problem, but also the first observational evidence for the Hawking radiation itself.

\section{acknowledgments}

This work was supported in part by the topical research program (2009 -T-1) of Asia Pacific Center for Theoretical Physics.

[1] A. G. Riess et al., Astron. J. 116, 1009 (1998).

[2] S. Perlmutter et al., Astroph. J. 517, 565 (1999).

[3] D. N. Spergel et al., arXiv:astro-ph/0603449 (2006). 
[4] M. Tegmark et al., Phys. Rev. D 74, 123507 (2006).

[5] P. J. E. Peebles and B. Ratra, Rev. Mod. Phys. 75, 559 (2003).

[6] R. R. Caldwell, R. Dave, and P. J. Steinhardt, Phys. Rev. Lett. 80, 1582 (1998).

[7] B. Ratra and P. J. E. Peebles, Phys. Rev. D 37, 3406 (1988).

[8] C. Armendariz-Picon, V. Mukhanov, and P. J. Steinhardt, Phys. Rev. Lett. 85, 4438 (2000).

[9] R. R. Caldwell, Phys. Lett. B 545, 23 (2002).

[10] S. Nojiri and S. D. Odintsov, General Relativity and Gravitation 38, 1285 (2006).

[11] A. Kamenshchik, U. Moschella, and V. Pasquier, Phys. Lett. B 511, 265 (2001).

[12] B. Feng, X. Wang, and X. Zhang, Phys. Lett. B 607, 35 (2005).

[13] H. Wei and R.-G. Cai, arXiv:0708.0884 (2007).

[14] P. Gough, arXiv:0709.0612 [astro-ph] (2007).

[15] M. Li, Phys. Lett. B 603, 1 (2004).

[16] A. G. Cohen, D. B. Kaplan, and A. E. Nelson, Phys. Rev. Lett. 82, 4971 (1999).

[17] R. Bousso, Rev. Mod. Phys. 74, 825 (2002).

[18] Y. Zeldovich, JETP Lett 6, 883 (1967).

[19] V. G. Gurzadyan and S.-S. Xue, Mod. Phys. Lett. A 18, 561 (2003).

[20] T. Padmanabhan, Gen. Rel. Grav. 40, 529 (2008).

[21] G. Mahajan, S. Sarkar, and T. Padmanabhan, Phys. Lett. B. 641, 6 (2006).

[22] S. G. Djorgovski and V. G. Gurzadyan, Nucl. Phys. Proc. Suppl. 173, 6 (2007).

[23] J. Gariel and G. L. Denmat, Classical and Quantum Gravity 16, 149 (1999).

[24] C. Barbachoux, J. Gariel, and G. le Denmat, Phys. Lett. B 658, 181 (2008).

[25] J. Gariel, G. Le Denmat, and C. Barbachoux, Phys. Lett. B 629, 1 (2005).

[26] T. Padmanabhan, Class. and Quantum Grav. 22, L107 (2005).

[27] R.-G. Cai, Phys. Lett. B 657, 228 (2007).

[28] Y. S. Myung and M.-G. Seo, arXiv:0803.2913 [gr-qc] (2008).

[29] J.-W. Lee, J. Lee, and H.-C. Kim, JCAP08(2007)005 (2007).

[30] J.-W. Lee, J. Lee, and H.-C. Kim, Proceedings of the National Institute for Mathematical Science 8, 1, arXiv:0709.0047 [hep-th] (2007).

[31] P. Gough, arXiv:0709.0612 [astro-ph] (2007).

[32] R. Horvat, arXiv:0711.4013 [gr-qc] (2007).

[33] A. J. M. Medved, arXiv:0802.1753 [hep-th] (2008).

[34] Z. Merali, Newscientist October 6, 16 (2007).

[35] R. Cowen, Science news 173, 74 (2008).

[36] S. W. Hawking, Comm. Math. Phys. 43, 199 (1975).

[37] L. Bombelli, R. K. Koul, J. Lee, and R. D. Sorkin, Phys. Rev. D 34, 373 (1986).

[38] J. Lee, H.-C. Kim, and J.-W. Lee, Phys. Lett. B 661, 67 (2007).

[39] R. H. Brandenberger, Rev. Mod. Phys. 57, 1 (1985).

[40] G. W. Gibbons and S. W. Hawking, Phys. Rev. D 15, 2738 (1977).

[41] A. Vilenkin, Nuclear Physics B 226, 527 (1983).

[42] E. Keski-Vakkuri and M. S. Sloth, Journal of Cosmology and Astroparticle Physics 2003, 001 (2003).

[43] R. Bousso, arXiv:hep-th/0205177 (2002).

[44] T. Jacobson, Phys. Rev. Lett. 75, 1260 (1995).

[45] Y. Gong, B. Wang, and A. Wang, JCAP 0701, 024 (2007).

[46] R.-G. Cai, L.-M. Cao, and Y.-P. Hu, arXiv:0809.1554 (2008).

[47] M. Srednicki, Phys. Rev. Lett. 71, 666 (1993).

[48] J. M. Maldacena, Adv. Theor. Math. Phys. 2, 231 (1998).

[49] H. Wei and S. N. Zhang, Phys. Rev. D 76, 063003 (2007).

[50] Y. S. Myung, Phys. Lett. B652, 223 (2007).

[51] M. Li, C. Lin, and Y. Wang, arXiv:astro-ph/0801.1407 (2008).

[52] S. Hawking, J. M. Maldacena, and A. Strominger, JHEP 05, 001 (2001).

[53] R. Müller and C. O. Lousto, Phys. Rev. D 52, 4512 (1995).

[54] M. Srednicki, Phys. Rev. Lett. 71, 666 (1993).

[55] M. A. Nielsen and I. L. Chuang, Quantum Computation and Quantum Information (Cambridge University Press, Cambridge, 2001).

[56] S. D. H. Hsu, Phys. Lett. B 594, 13 (2004).

[57] M. D. Pollock and T. P. Singh, Classical and Quantum Gravity 6, 901 (1989).

[58] X. Zhang and F.-Q. Wu, arXiv:astro-ph/0701405 (2007).

[59] Q.-G. Huang and M. Li, JCAP 08, 013 (2004).

[60] Q.-G. Huang and Y. Gong, JCAP 2004, 006 (2004).

[61] J.-Q. Xia, H. Li, G.-B. Zhao, and X. Zhang, arXiv:0708.1111 (2007).

[62] G.-B. Zhao et al., Phys. Lett. B 648, 8 (2007).

[63] T. Banks and W. Fischler, astro-ph/0307459 (2003).

[64] B. Wang and E. Abdalla, Phys. Rev. D 69, 104014 (2004).

[65] N. Kaloper, M. Kleban, and L. Sorbo, Phys. Lett. B600, 7 (2004). 
[66] S. Dodelson and L. Hui, Phys. Rev. Lett. 91, 131301 (2003).

[67] A. R. Liddle and S. M. Leach, Phys. Rev. D 68, 103503 (2003).

[68] H.-C. Kim, J.-W. Lee, and J. Lee, JCAP 08, 035 (2008).

[69] E. W. Kolb and M. Turner, The Early Universe (Addison-Wesley, New York, 1990).

[70] K. Greisen, Phys. Rev. Lett. 16, 748 (1966).

[71] T. Stanev, C. R. Physique 5, 453 (2004).

[72] F. W. Stecker, Phys. Rev. Lett. 21, 1016 (1968).

[73] R. Protheroe and P. Johnson, Astroparticle Physics 4, (February 1996).

[74] R. U. Abbasi et al., Phys. Rev. Lett. 100, 101101 (2008).

[75] C. Beck and M. C. Mackey, Phys. Lett. B 605, 295 (2005).

[76] L. R. W. Abramo, R. H. Brandenberger, and V. F. Mukhanov, Phys. Rev. D 56, 3248 (1997).

[77] A. D. Linde, arXiv:hep-th/0503203 (2005).

78] F. Melia, MNRAS 382, 1917 (2007).

[79] B. W. Yungui Gong and A. Wang, JCAP 2007, 024 (2007).

[80] W. G. Unruh, Phys. Rev. Lett. 46, 1351 (1981).

[81] T. A. Jacobson and G. E. Volovik, Phys. Rev. D 58, 064021 (1998).

[82] T. G. Philbin et al., Science 319, 1367 (2008). 